\title{
Antioxidant capacity and phenolic acids of virgin coconut oil.
}

\begin{abstract}
The antioxidant properties of virgin coconut oil produced through chilling and fermentation were investigated and compared with refined, bleached and deodorized coconut oil. Virgin coconut oil showed better antioxidant capacity than refined, bleached and deodorized coconut oil. The virgin coconut oil produced through the fermentation method had the strongest scavenging effect on 1,1-diphenyl-2-picrylhydrazyl and the highest antioxidant activity based on the $\beta$-carotene-linoleate bleaching method. However, virgin coconut oil obtained through the chilling method had the highest reducing power. The major phenolic acids detected were ferulic acid and p-coumaric acid. Very high correlations were found between the total phenolic content and scavenging activity $(\mathrm{r}=0.91)$, and between the total phenolic content and reducing power $(\mathrm{r}=0.96)$. There was also a high correlation between total phenolic acids and $\beta$-carotene bleaching activity. The study indicated that the contribution of antioxidant capacity in virgin coconut oil could be due to phenolic compounds.
\end{abstract}

Keyword: Antioxidant activity; Reducing power; Scavenging effect; Total phenolics. 